\title{
Spatial Distribution of Adolescents with Sexually Transmitted Infections Diagnosed in the Pediatric Emergency Departments of Washington, DC
}

\author{
Shilpa Patel ${ }^{\star 1}$, Lisa Tuchman², Katie Hayes ${ }^{3}$, Gia Badolato ${ }^{1}$, Stephen J. Teach ${ }^{1}$ and Monika \\ K. Goyal ${ }^{1}$
}

${ }^{1}$ Division of Emergency Medicine, Children's National Medical Center, Washington DC, DC, USA; 'Division of Adolescent Medicine, Children's National Medical Center, Washington DC, DC, USA; ${ }^{3}$ Division of Emergency Medicine, Children's Hospital of Philadelphia, Philadelphia, PA, USA

\section{Objective}

(1) To describe the spatial distribution of adolescents with EDdiagnosed STIs in a large urban area with a high prevalence of STI

(2) To compare census block groups and identify "hot spots" of STI.

\section{Introduction}

Utilization of local surveillance data has been shown to help risk stratify patients presenting to the emergency department presenting with GAS pharyngitis or meningitis. $(1,2)$ Adolescents frequently present to the emergency department (ED) with symptoms that may be associated with a sexually transmitted infection (STI). (3)

When ED providers perceive high local rates of STI and low rates of follow-up, empiric treatment is considered. This strategy may result in unnecessary treatment. Knowledge of the local spatial distribution of adolescents with STIs diagnosed in local pediatric emergency departments EDs may enhance risk stratification and allow targeted testing and/or treatment among future ED patients in whom STI is considered.

\section{Methods}

We performed a retrospective cross-sectional medical record review of all visits made by District residents aged 13-19 years old to the two large urban pediatric EDs in Washington, DC in 2012 and abstracted demographic and visit data, including STI testing results for gonorrhea and chlamydia. Multivariable logistic regression was utilized to compare odds of testing positive for STI between geographic regions. Geospatial statistical analyses were performed using ArcMap 10.1 tools, Hot Spot Analysis and Spatial Autocorrelation (Moran's I).

\section{Results}

Of 1002 adolescents tested for STIs, $6.8 \%$ tested positive for gonorrhea, $18.6 \%$ tested positive for chlamydia, $22.7 \%$ tested positive for either gonorrhea or chlamydia and $2.9 \%$ tested positive for coinfection. The mean age of patients with STIs was 16.9 years (SD 1.5 ); $78.4 \%$ were female. Of the 227 patients with $\geq 1$ positive test, $95 \%$ of their addresses were successfully geocoded. Hot Spot Analysis indicated statistically significant clusters of STI cases (figure 1.) Spatial autocorrelation (Moran's I) demonstrated that the distribution of cases was not random ( $\mathrm{z}$-score 13.3, p value $<0.05$ ). The odds of adolescent patients from DC Ward 6 testing positive for chlamydia was twice that of DC Ward 7(aOR 2.07; 95\% CI 1.07-3.98).

\section{Conclusions}

Geographic analyses identified areas with significantly higher cases of ED-diagnosed STIs. Modeling real time surveillance in conjunction with other clinical information readily available in the electronic medical record may improve prediction of STIs in adolescents presenting to the ED, decrease empiric treatment, and allow for targeted public health prevention. Incorporation of city-wide data could improve our ability to identify clusters of infection.

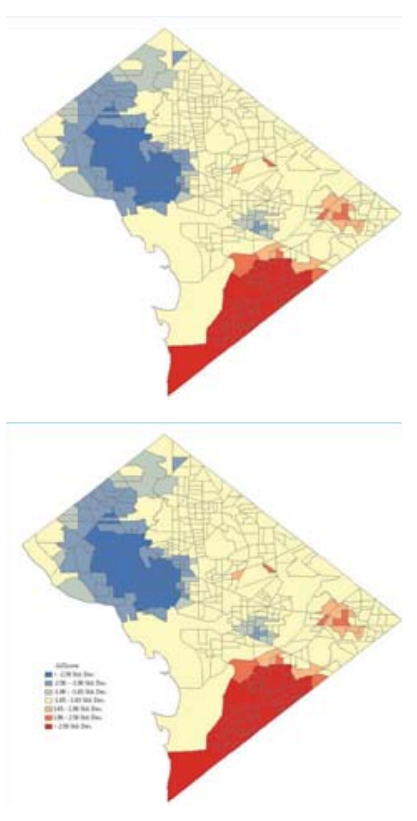

Figure 1. Hot spot analysis of ED-diagnosed STI cases by census block group $(n=233)$

\section{Keywords}

sexually transmitted infection; surveillance; emergency department

\section{Acknowledgments}

We would like to thank Drs. Kristen Breslin and James Chamberlain for their contribution to study conception and design, Prof. Dante Verme of George Washington University for assistance with GIS and the DC GIS program and www.census.gov for providing the base maps.

\section{References}

(1)Fine AM, Nizet V, Mandl KD. Improved diagnostic accuracy of group A streptococcal pharyngitis with use of real-time biosurveillance. Ann Intern Med. 2011 Sep 20;155(6):345-52.

(2)Fine AM, Nigrovic LE, Reis BY, Cook EF, Mandl KD. Linking surveillance to action: incorporation of real-time regional data into a medical decision rule. Am Med Inform Assoc. 2007 Mar-Apr; 14(2):206-11.

(3)Goyal M, Hayes K, Mollen C. Sexually transmitted infection prevalence in symptomatic adolescent emergency department patients. Pediatric Emerg Care. 2010 Dec;28(12):1277-80.

\section{*Shilpa Patel}

E-mail: shilpapateldas@gmail.com 